\title{
PERCEPCIÓN DE LA CALIDAD DEL SERVICIO DE TRANSPORTE URBANO EN LA CIUDAD DE FLORENCIA
}

\author{
Yaqueline Llanos Burgos ${ }^{1}$ \\ Diana Duperly Llanos Burgos ${ }^{2}$ \\ Iraide Molina Peralta ${ }^{3}$
}

Recibido: 27 de abril de 2013

Aceptado: 12 de agosto de 2013

\section{Resumen}

La calidad del servicio es un aspecto importante dentro de una organización, para que ésta se mantenga en el mercado. En agosto de 2012 se realizó una investigación que colaboró en conocer la percepción de los usuarios del servicio urbano en la ciudad de Florencia, con el fin de determinar cómo se puede mesurar la calidad del mismo.

Para el desarrollo de la investigación se hizo presencia en los sectores que conforman el perímetro urbano de la ciudad, (barrios normales y subnormales del Municipio), usando una muestra de 384 personas todas usuarios del servicio urbano, obteniendo como resultado una insatisfacción del $76 \%$ de la población, que obedece a diferentes razones como es la falta de asignación de rutas para cubrimiento del servicio en todos los sectores, el mal estado de las vías, el deterioro y el desaseo de los vehículos que prestan el servicio. Sumado a ello, la atención poco amable que prestan los conductores de los vehículos de servicio colectivo y los largos tiempos de espera a que se someten los usuarios. El moto taxi, ha sido tomado como transporte alterno el cual es un servicio no autorizado legalmente y en la actualidad opera de manera masiva.

Palabras claves: pservicio, transporte, calidad, movilidad, percepción, servicio urbano.

\footnotetext{
${ }^{1}$ Candidata a obtener el título en Administración de Empresas, jackellanos@gmail.com

2 Estudiante de Administración de empresas, diana2812@gmail.com

${ }^{3}$ Candidata Master en Dirección estratégica de mercadeo, Especialista en Gerencia estratégica de mercadeo,

Administradora de empresas, Docente ECACEN CEAD Florencia, Iraide.molina@unad.edu.co
} 


\section{PERCEPTION OF SERVICE QUALITY IN URBAN TRANSPORT IN FLORENCIA CITY}

\section{Abstract}

The quality of service is important within an organization, to keep it on the market. In August 2012 was carried out an investigation which it collaborates in the perception of the users of the urban service in the city of Florencia, in order to determine how it can measure the quality of it.

For the development of the research was presence in the sectors that make up the urban perimeter, (normal and subnormal neighborhoods of the municipality), using a sample of 384 people all users of the urban service, obtaining as a result a dissatisfaction of the $76 \%$ of the population, which is due to different reasons as is the lack of allocation of routes for coverage of the service in all sectors, the poor condition of the rail tracks, the deterioration and the messiness of the vehicles that provide the service. In addition to this, the attention little friendly providing by drivers of the vehicles of collective service and long waiting times that the users are subjected. The mototaxi, has been taken as alternate transport which is a service that is not legally authorized and currently operates on a massive scale.

Keywords: Service, Transportation, quality, mobility, perception, urban service.

\section{Introducción}

La calidad del servicio de transporte público urbano está dada por la capacidad de satisfacer los deseos de los usuarios que demandan el servicio. Luego lo deseable es una mejor calidad del servicio para así obtener una mayor satisfacción del usuario. Esta «satisfacción» del usuario se puede medir de acuerdo con los atributos que éste considera necesarios y relevantes al momento de acceder al servicio. Para Schiffman y Kanuk (2005), «los consumidores evalúan la calidad de un producto o servicio, tomando señales de información que han llegado a asociar con dicho producto, refiriéndose a características intrínsecas del producto o servicio mismo, en tanto que otras son de carácter extrínseco» (p. 188).

El sistema de transporte urbano permite a los ciudadanos movilizarse desde sus hogares a sus lugares de trabajo, recreación, diversión, cultura, deporte, en fin, participar en las actividades urbanas características de las ciudades. Esto hace que el transporte se constituya en un derecho adquirido por la población y que el Estado se convierta en garante de este servicio para contribuir con la calidad de vida de los habitantes de cada localidad'1.

El objetivo del Transporte urbano no debe ser simplemente movilizar a los usuarios, sino aumentar, el bienestar de los habitantes, de esta forma, para las empresas de transporte urbano, Palermo y Villanueva (2005) «un incremento en la satisfacción del cliente se traduce en: a) retener los usuarios cautivos, b) aumentar el uso del sistema atrayendo nuevos usuarios y c) me-

${ }^{4}$ Tomado de Políticas y calidad de servicio del transporte público. Agosto de 2012. http://www.voxlocalis.net/revistas/num14/doc/docviii1.pdf 
jorar la imagen pública» (p. 1). Siendo el usuario la prioridad en el éxito del sistema, es muy importante el considerar su percepción sobre la calidad del servicio brindado. Según Kotler y Armstrong (2003) «la percepción es el proceso por el que las personas seleccionan, organizan e interpretan la información para formarse una imagen inteligible del mundo» (p. 202); por lo anterior aspectos como la seguridad, accesibilidad, movilidad, comodidad, trato personalizado y comunicación al cliente son de vital importancia para determinar percepción de la excelencia del servicio de transportes.

En la Ciudad de Florencia, desde el año 2009 se vienen presentando una serie de inconvenientes, relacionados con el Transporte Público Colectivo, donde los propietarios y conductores de los vehículos que prestan el servicio han realizado «paros» y «planes tortuga», para manifestar su inconformidad ante el gobierno local, y a la vez reclamar una mejor organización y control al transporte ilegal, ya que se sienten afectados en la parte económica debido a que lo producido permite su subsistencia descuidando el mantenimiento de sus vehículos, afectando enormemente la prestación del servicio urbano en la Ciudad, es de anotar que a la fecha en Florencia existen cuatro (4) empresas de Transporte urbano, denominadas; Cootransflorencia, Coomotorflorencia, Circular Florencia y Cootranscaqueta, que movilizan 133 vehículos de servicio urbano en toda la ciudad 2 .

Ante la situación descrita, se consideró importante investigar a cerca de la percepción que tienen los usuarios del servicio urbano, y las razones por las cuales acceden o no al servicio. De esta forma el objetivo general de la investigación corresponde a «Identificar cuál es la percepción del usuario sobre la calidad del servicio de transporte urbano en la Ciudad de Florencia». Con el fin de definir este objetivo y los objetivos específicos de la investigación, que corresponden a: Conocer bajo qué condiciones técnicas se presta el servicio de transporte urbano en la ciudad de Florencia, Conocer la frecuencia con la que el usuario accede al servicio urbano de la Ciudad de Florencia, Investigar si el usuario de Transporte urbano prefiere hacer uso de otras alternativas de transporte, establecer si son suficientes las rutas, frecuencias y horarios del servicio urbano en la Ciudad de Florencia, determinar cuál es el principal inconveniente para trasladarse de un lugar a otro en la Ciudad de Florencia, en vehículos de servicio urbano, determinar si el usuario de transporte urbano de la Ciudad de Florencia, es un usuario satisfecho.

El trabajo investigativo es de gran utilidad para el organismo de tránsito de la ciudad, y para toda entidad que tenga relación con el transporte urbano en la ciudad, lo mismo que para la universidad, teniendo en cuenta que contiene información real y estadísticas que permiten conocer las razones de insatisfacción del usuario ante el servicio de transporte urbano y las posibles estrategias o planes de mejoramiento al problema.

\section{Método}

El proceso investigativo se desarrolló como una investigación cuantitativa, descriptiva, según Malhotra, (1997) «la investigación descriptiva es el tipo de investigación concluyente que tiene como objetivo principal la descripción de algo, generalmente las características o funciones del problema en cuestión» (p. 90), de acuerdo a la problemática y los objetivos planteados con el desarrollo de la investigación se buscaba detallar o retratar las características que inciden en la percepción de la calidad del servicio de transporte, obteniéndose así estadísticas e información numérica conducente a la solución y logro del problema y objetivos respectivamente. 
La técnica de recolección de información utilizada para la obtención de datos fue la encuesta personal, que contiene 26 preguntas con opciones de respuesta cerradas, dirigidas a usuarios del servicio de transporte urbano. El trabajo de campo se realizó sobre el objeto de estudio que corresponde a la población que hace uso del servicio de transporte urbano en la ciudad, ya que de acuerdo a Beverly (2004) Antes de poder atender debidamente a tus clientes, debes estar en posibilidad de identificarlos (p.28); obteniendo la información por medio de las encuestas realizadas y que fueron dirigidas a una población infinita determinándose una muestra de 384 personas (fuente primaria).

De igual manera como fuente secundaria se obtuvo información sobre los vehículos de servicio público urbano que actualmente prestan el servicio en la Ciudad, las normas que rigen el funcionamiento del transporte colectivo de pasajeros, el control, las condiciones de seguridad, rutas, horarios, y tarifas, en entidades como; las empresas transportadoras y la Secretaria de Transito y Movilidad de Florencia. Para el procesamiento de la información se hizo uso del programa Excel que fue usado para la respectiva tabulación, graficación, interpretación de la información para posteriormente analizarla.

\section{Resultados}

Los resultados obtenidos en el desarrollo de la investigación, precisan en la solución de la problemática y alcance de objetivos, entre esto se obtuvo:

Figura 1. Estado de mantenimiento de los vehículos

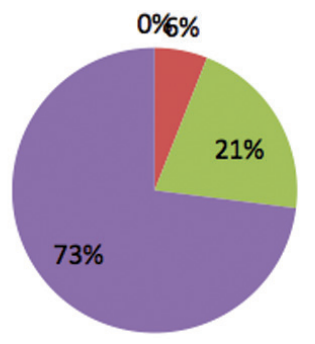

Excelente

mbueno

" pésimo

deteriorado

Fuente. Encuestados, junio 2012
El 73\% de la población considera que los vehículos con los que se presta el servicio de transporte público urbano en la ciudad de Florencia se encuentra deteriorado, el $21 \%$ considera que el estado de los vehículos es pésimo y el $6 \%$ considera que los vehículos se encuentran en buen estado.

Figura 2. Estado de las sillas y demás elementos internos de los vehículos

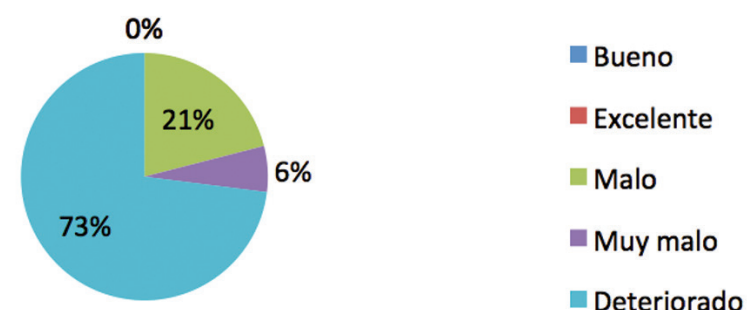

Fuente. Encuestados, junio 2012

El $73 \%$ de la población considera que las sillas y demás elementos internos de los vehículos con los que se presta el servicio de transporte público urbano en la ciudad de Florencia se encuentra deteriorado, el $21 \%$ de la población considera que se encuentra en estado Malo y el $6 \%$ de la población considera que se encuentran en buen estado. Se entiende una gran insatisfacción por parte de la población.

Figura 3. Frecuencia con que hace uso del servicio

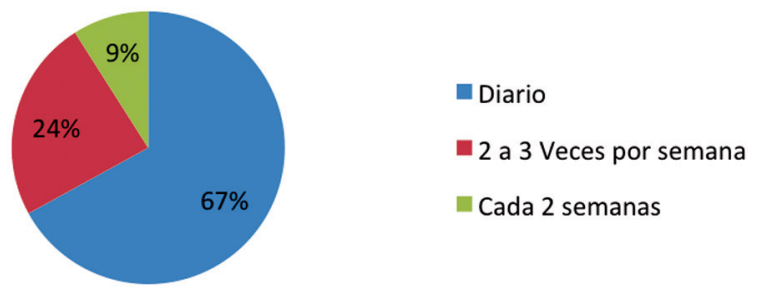

Fuente. Encuestados, junio 2012

Un $67 \%$ de la población hace uso de servicio urbano diariamente, un $24 \%$ lo hace de 2 a 5 veces por semana y solo un $9 \%$ usa este servicio cada dos semanas. Son muchas las personas que acceden al servicio urbano, y en su mayoría lo hacen diariamente, esto va ligado a la necesidad del usuario, de acuerdo a su actividad diaria. 
Un $76 \%$ de la población considera que los vehículos que prestan el servicio urbano de pasajeros, se encuentran desaseados, mientras

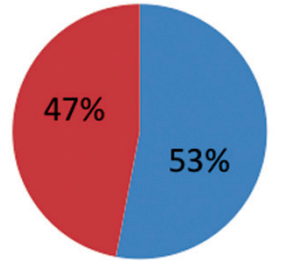

- 15 minutos

- 30 minutos

Fuente. Encuestados, junio 2012

Un 53\% de la población ha manifestado que el tiempo de espera para tomar este servicio es de 15 minutos y un $47 \%$ ha manifestado que deben esperar 30 minutos para acceder al servicio.

Figura 5. Dificultades para movilizarse en el servicio urbano

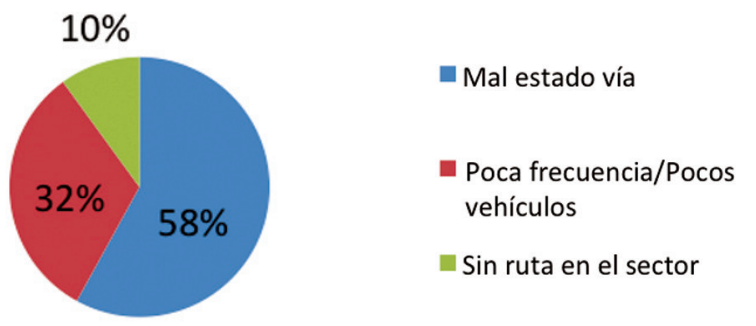

Fuente. Encuestados, junio 2012

La dificultad que es considerada la más frecuente para trasladarse haciendo uso del servicio de transporte urbano colectivo es el mal estado de las vías con un $58 \%$; la poca frecuencia/pocos vehículos con un $32 \%$, de igual manera un $10 \%$ de la población considera que el inconveniente es que la ruta no pasa por el sector.

Figura 6. Condiciones de aseo de los vehículos

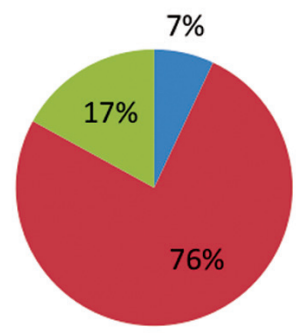

Aseado

Desaseado

Un poco aseado que un $17 \%$ consideran que los vehículos se encuentran un poco aseados y un $7 \%$ consideran que estos vehículos se encuentran aseados.

Figura 7. Alternativa de transporte más utilizada

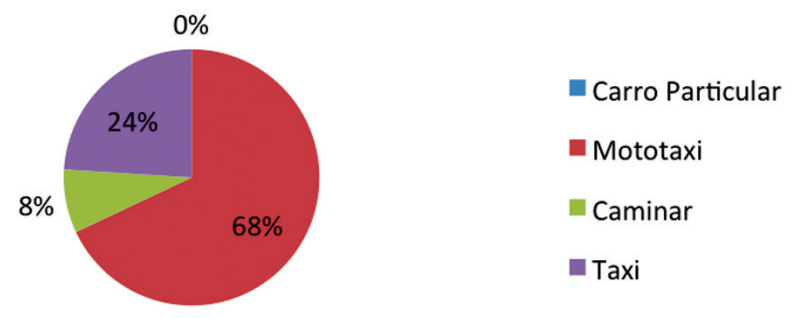

Fuente. Encuestados, junio 2012

Existen causas que llevan al usuario a tomar la decisión de acudir a otra alternativa de transporte diferente al servicio colectivo para movilizarse a cumplir sus obligaciones diarias, aunque son conscientes de poner en riesgo su integridad física en algunas de ellas, es así que el $68 \%$ de la población tiene como opción de transporte el mototaxismo, el $24 \%$ usa como opción de transporte el servicio de taxi y el $8 \%$ decide caminar.

Figura 8. Aspectos positivos de la alternativa de transporte escogida

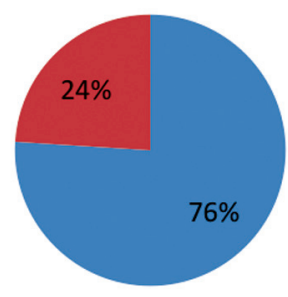

Rapidez

- Servicio económico

Fuente. Encuestados, junio 2012

El $76 \%$ de la población que tiene como alternativa de transporte del mototaxismo considera que es la rapidez de este servicio lo que motiva a hacer uso de él. El 24\% de la población considera que es un servicio económico, comparado con el taxi, mas no con el servicio de transporte urbano suministrado por los buses. 
Figura 9. Aspectos negativos que posee la alternativa de transporte escogida

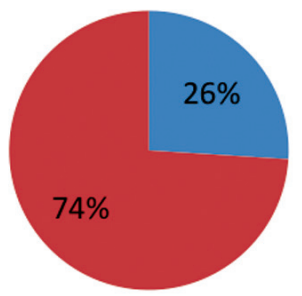

inseguridad

Riesgo Accidente

Fuente. Encuestados, junio 2012

De la población que ha escogido como alternativa de transporte el mototaxismo, el $74 \%$ considera que existe riesgo de accidentes y el $26 \%$ considera que el uso de este servicio ilegal ocasiona inseguridad. Pese a esto, hacen uso del mismo.

Figura 10. Ruta urbana más utilizada

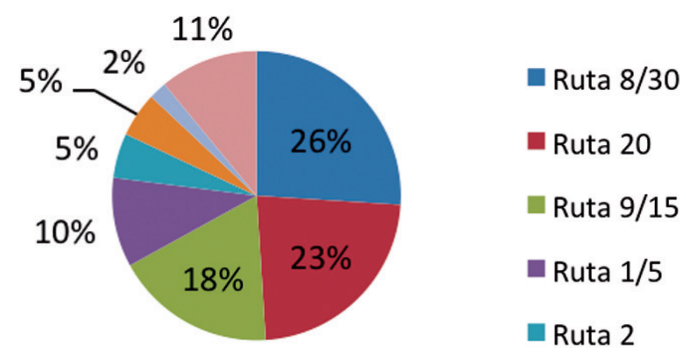

Fuente. Encuestados, junio 2012

De las 9 rutas autorizadas por el organismo de transito de la ciudad, la ruta $8 / 30$ es la más usada por un $26 \%$, siguiendo la ruta 20 por un $23 \%$ y por un $18 \%$ la ruta $9 / 15$. La ruta $8 / 30$ tiene gran cubrimiento del servicio en la comuna norte, la ruta 20 y la 2 tienen su mayor cubrimiento del servicio en la comuna sur. Con el fin de complementar la información sobre rutas de acceso se indagó sobre la calificación de las rutas asignadas; obteniendo como resultado que de las 9 rutas un $48 \%$ de la población encuestada considera que las rutas asignadas para la ciudad son nada satisfactorias, un $30 \%$ considera que las rutas asignadas son poco satisfactorias y un $22 \%$ considera que son suficientes.
Figura 11. Grado de satisfacción del usuario del servicio de transporte colectivo

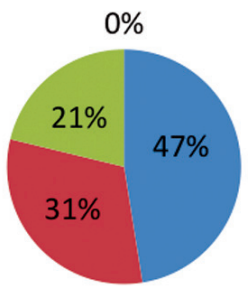

Nada satisfecho

poco satisfecho

satisfecho

muy satisfecho

Fuente. Encuestados, junio 2012

El $47 \%$ de la población manifiesta que se sienten nada satisfechos con el servicio ofrecido por el sistema de transporte colectivo en la ciudad, un $31 \%$ considera que se siente poco satisfecho y un $21 \%$ se siente satisfecho con este servicio. Al evidenciar los resultados se obtienen un $78 \%$ (sumatoria nada satisfecho y poco satisfecho) de insatisfacción con el servicio, factor que se debe de analizar por parte de las empresas transportadoras para lograr posicionarse en los usuarios del servicio. Sumado a lo anterior cuando se les preguntó sobre el tiempo de espera del servicio colectivo, el $47 \%$ de los encuestados ha manifestado que el servicio es retrasado y un $40 \%$ lo considera demorado, el $13 \%$ restante considera que es adecuado. Así mismo, se indago sobre la atención al usuario por parte del conductor del vehículo y como resultados se obtuvo que el $49 \%$ de la población considera que se atendió de manera amable por parte del conductor del vehículo, un 33\% considera que es atendido de manera descortés y un $18 \%$ considera que es atendido de una forma nada amable por parte del conductor del vehículo.

Figura 12. Norma de tránsito más infringida por los conductores

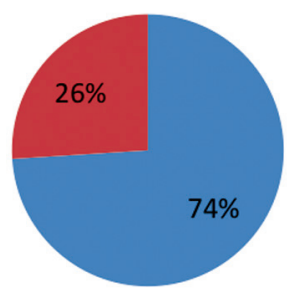

$$
\begin{aligned}
& \text { Descarga y recoger } \\
& \text { pasajeros en sitios no } \\
& \text { permitidos } \\
& \text { estacionar en sitios } \\
& \text { prohibidos }
\end{aligned}
$$


De igual manera un $74 \%$ de la población considera que los conductores de estos vehículos incurren en una infracción de tránsito denominada descargar y recoger pasajeros en sitio que no son autorizados, a la vez un $28 \%$ manifiesta que los conductores incurren otra infracción que corresponde a estacionar en sitio no permitidos. De igual y para complementar la percepción sobre el respeto a la normatividad de tránsito por parte de los conductores se evidencia que el $72 \%$ de la población considera que los conductores de los vehículos de servicio urbano colectivo irrespetan las normas de tránsito, y un $28 \%$ consideran que no existe infracción a las normas de tránsito por parte de ellos.

Figura 13. Sugerencias para el mejoramiento del servicio de transporte urbano en la ciudad de Florencia

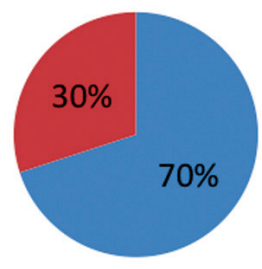

Incrementar vehículos

Más rutas

Fuente. Encuestados, junio 2012

El $70 \%$ de la población sugiere para el mejoramiento del servicio de transporte urbano el incremento de vehículos para el cubrimiento de rutas, y el $30 \%$ de la población sugiere la asignación de más rutas en la ciudad. Este aspecto debe estudiarse teniendo en cuenta factores externos en la prestación y uso de servicio.

\section{Discusión}

Es de resaltar que las personas encuestadas corresponden a usuarios del servicio urbano colectivo de la ciudad, entendidos por Domínguez (2006) como «los intermediarios que directamente tienen relación con la empresa y hacia los cuales deben dirigirse las acciones estratégicas para que se manifieste un valor agregado perceptible y una calidad de servicio que verdaderamente establezca una diferencia» (p.5) entre ellos se tuvieron en cuenta: amas de casa, comerciantes, estudiantes, empleados públicos y particulares, trabajadores independientes; quienes a pesar de ser usuarios de este servicio, no tienen como única opción de transporte el servicio colectivo, sino que acuden a otras alternativas de transporte. Teniendo en cuenta la calidad percibida de los servicios, según Shiffman y Kanuk (2005) «la evaluación que realiza el consumidor acerca de la calidad de los servicios depende de la magnitud y la dirección de la brecha entre sus expectativas sobre el servicio y la evaluación (percepción) del servicio que realmente reciba» (p. 189), esto se puede constatar con los resultados obtenidos sobre la percepción en la calidad de servicio de transporte urbano, en la ciudad de Florencia, ya que al evaluarlo, las condiciones actuales que se esperan en la prestación de servicios no superan las expectativas que éstos poseen no solo por los factores internos en la prestación de servicio sino también de los externos; es así que actualmente por parte del usuario del servicio existe insatisfacción en cuanto a condiciones de aseo de los vehículos que prestan el servicio, a cubrimiento de rutas por parte del servicio urbano colectivo de pasajeros, al mantenimiento de los vehículos lo cual no es favorable para el sector; sumado a lo anterior se tiene que la frecuencia del tiempo establecido por el organismo de transito según la autorización de rutas, frecuencias y horarios es de 6 minutos, donde cada conductor debe tomarse un tiempo de recorrido por ruta entre 67 y 94 minutos de acuerdo a la ruta que se encuentre cubriendo, lo anterior no se está cumpliendo a cabalidad, no siempre por negligencia de quienes prestan el servicio, sino también por factores externos como son los inconvenientes que se presentan para la movilización en el servicio urbano, específicamente el deterioro de la vías, este aspecto ha contribuido a que el uso de este servicio disminuya, ya que transitar por vías en deterioro ocasiona tardanza en el cubrimiento de la rutas, y los tiempo de espera son más extendidos. 
Por otra parte se evidencia que la población ha optado por tener otras alternativas de transporte tanto legal como ilegal como es el caso del mototaxismo. Ante tal situación es inquietante el hecho de tener conocimiento sobre las medidas que las autoridades locales pertinentes tomen a cerca de este fenómeno, que se ha incrementado cada día más y que es evidente que este fortalecimiento del servicio de mototaxi obedece a las falencias en la prestación del servicio de transporte colectivo. Por lo tanto las autoridades deben tomar medidas no tanto restrictivas para contrarrestar el mototaxismo sino tendientes al mejoramiento del servicio de transporte colectivo en la Ciudad de tal manera que el usuario reciba un buen servicio sintiéndose satisfecho del mismo y no se vea obligado a tomar un transporte alterno en el cual ponga en riesgo su integridad; así mismo el uso de taxis, de vehículos particulares se han venido incrementando en la ciudad y esto conlleva a que el transporte urbano pierda participación en el mercado y a desmejorar la intención de uso del servicio.

De acuerdo a lo anterior, se evidencian debilidades que presenta la prestación del servicio urbano en la ciudad de Florencia, por lo tanto, se requiere que la presente investigación, sea fuente de información para la Secretaria de Tránsito y Transporte del municipio de Florencia, con el fin de que éste organismo logre identificar estrategias que colaboren en el mejoramiento de las prestación del servicio, tanto para los usuarios como también para las empresas que proveen dicho servicio de transporte.

\section{Conclusiones}

La percepción del usuario sobre la calidad del servicio de transporte colectivo en la ciudad de Florencia, no es satisfactoria, situación que obedece a diferentes factores, como son los tiempos de espera, las rutas establecidas, el estado de mantenimiento de los vehículos, la atención por parte del conductor, las condiciones de aseo; factores que influyen al momento de formar un concepto por parte del usuario y que hacen que este haga uso de otras alternativas de transporte como el transporte ilegal denominado Mototaxismo y que pese a conocer los riesgos que genera el uso de estas motocicletas, la usan como opción alterna, ya que le ofrece unas ventajas como llegar rápido a su destino.

Pese a que la percepción que tienen los usuarios del servicio colectivo en la ciudad, sobre la calidad de este, no es la mejor, muchos de ellos se ven obligados a hacer uso del servicio por «necesidad» y de esta forma se someten a las incomodidades que actualmente el servicio posee y que ya fueron mencionadas, como son los largos tiempos de espera, desaseo, inseguridad por deterioro, irrespeto a las normas de tránsito, mal estado de las vías, buscando una solución de transporte en el mototaxismo, que aunque no ofrece las condiciones mínimas de seguridad para su movilización, le ofrece ventajas como llegar rápido a su destino, y en ocasiones por menos precio.

El problema de transporte en la ciudad de Florencia, radica en gran parte en el mal estado de los vehículos usados para prestar el servicio, situación que debe ser atendida por las empresas prestadoras del servicio. Otro aspecto relevante es la falta de regulación de tiempos y frecuencias como también las rutas establecidas que no son suficientes para el cubrimiento del servicio en la ciudad, ante lo cual los entes de control deben realizar una supervisión especial y exigir cumplimento de rutas, frecuencia y horarios.

De otro lado el mal estado de las vías y un parque automotor deteriorado hacen parte de las causales de insatisfacción y mal servicio a la comunidad. Sumado a lo anterior, el uso del transporte alterno «mototaxismo», que aunque ilegal e inseguro, ofrece al usuario un servicio rápido y económico, como solución al problema de transporte. 
El resultado de insatisfacción obtenido como respuesta al objetivo de esta investigación, se debe al mal estado de los vehículos $(73 \%)$, deficiencia en el cubrimiento de rutas (87\%), mal estado de las vías $(58 \%)$, entre otros aspectos ya mencionados, que indican el no cumplimiento de las determinantes de la calidad del servicio anotadas en el marco teórico, como la seguridad, la movilidad, comunicación, acceso, confiabilidad, que son vitales en la prestación del servicio; y relevantes al momento de determinar la calidad del mismo.

\section{Referencias}

Albrecht, K. Bradford, L. (1999). La excelencia en el servicio. Bogotá: ER. Editores.

Alcaldía de Florencia. Plan de ordenamiento territorial Municipio de Florencia 2000. (2000).

Recuperado de http://www.florencia-caqueta.gov.co/planeacion.shtml?apc=p1|1--\&x=2337201

Beverly R. J.L. (2004). Servicio al cliente. México: Thomson, Coren, S. (2001). Sensación y percepción. México: McGraw Hill.
Departamento Administrativo Nacional de Estadística. Perfil Municipal de Florencia -

Censo 2005.

Domínguez, H. (2006). El servicio invisible: fundamento de un buen servicio al cliente. Bogotá: Universidad Sergio Arboleda. Ecoe ediciones.

Kotler, P. (2002). Dirección de marketing. Conceptos esenciales. México: Pearson Educación.

Kotler, P. y Armstrong G. (2003). Fundamentos de Marketing. $6^{\circ}$ Edición, Ed. México: Pearson Prentice Hall.

Malhotra, N.k. (1997). Investigación de mercados. Un enfoque práctico. 2. Edición. Ed. México: Prentice Hall.

Palermo, K. y Villanueva, J.. (2005). Percepción del usuario de transporte colectivo sobre la calidad del servicio ofrecido por la AMA. Universidad Puerto Rico -Mayagüez. Recuperado de http://uprati.uprm.edu/interns/ group1 final reports/jaritcely.pdf

Schiffman, G. Léon y Kanuk, L. Leslie (2001). Percepción del Consumidor, en Comportamiento del Consumidor. Pp: 122-155. México: Prentice Hall. 